\title{
Recent advances of the OIV working group on oenological tannins in the study of the functionalities of oenological tannins
}

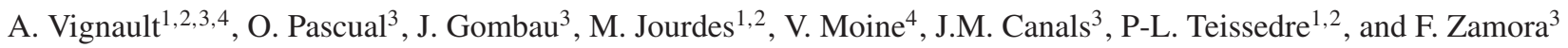 \\ ${ }^{1}$ Université de Bordeaux, Unité de recherche Enologie, EA 4577, USC 1366 INRA, ISVV, 33882 Villenave d'Ornon cedex, France \\ ${ }^{2}$ INRA, ISVV, USC 1366 Enologie, 33140 Villenave d'Ornon, France \\ ${ }^{3}$ Departament de Bioquímica i Biotecnologia, Facultat d'Enologia de Tarragona, Universitat Rovira i Virgili, C/Marcel.li Domingo 1, \\ 43007 Tarragona, Spain \\ ${ }^{4}$ Laffort, 11 rue Aristide Bergès, 33270 Floirac, France
}

\begin{abstract}
This communication actualizes the status of the research of the OIV working group on oenological tannins with the purpose of deepening the knowledge of the mechanisms and functionalities of oenological tannins in winemaking. The obtained results confirm that oenological tannins really exert an effect as copigments and that they really protect the wine color against the negative effect of the enzyme laccase present in grapes affected by the infection of Botrytis cinerea.
\end{abstract}

\section{Introduction}

The use of oenological tannins is common practice in winemaking. However, nowadays this use is only authorized by the OIV to facilitate the clarification of wines and musts [1]. Nevertheless, it is unquestionable that oenological tannins are also used for many other purposes such as to protect wines against oxidation, to improve wine structure and mouthfeel or to stabilize the color of red wines mainly [2]. Furthermore, under the term oenological tannins different families of compounds with different botanical origins and chemical composition are included. Specifically, oenological tannins include hydrolysable tannins (ellagitannins from oak or chestnut and gallotannins from gall nuts or tara) and condensed tannins (proanthocyanidins from grape seeds and skins, and from other plant origins such quebracho and mimosa) [3]. For all these reasons the OIV began a few years ago a deep reflection on the oenological tannins and created a working group to determine their chemical characterization and possible functionalities given the wide range of commercial tannins present in the market.

The main results of this research were presented last year at the meeting of the OIV Technology Commission in Paris and at the 41st Congress of the OIV in Uruguay [4]. These results confirm that oenological tannins protect wine against oxidation because they exert antioxidant capacity [3], they consume directly oxygen [5] and they inhibit laccase activity [6]. Moreover, they can improve and stabilize the color of red wines since they act as copigments [7-9]. As a fruit of these results the OIV resolutions Oeno-Techno 17-612 [10] and Oeno-Techno $17-613$ [11], now in step 7, propose to include two new objectives of the use of tannins in must and wine that will be probably approved in the next $17^{\text {th }}$ General Assembly of OIV. Specifically, these two objectives are:

- to contribute to the antioxidant protection of components of the must and wine

- to promote the expression, stabilization and preservation of color

The present communication actualizes the status of the research of the OIV working group on oenological tannins with the purpose of deepening the knowledge of the mechanisms and functionalities of oenological tannins in winemaking. This communication is in fact a summary of previously published articles [6-9].

\section{Materials and methods}

\subsection{Commercial tannins}

Thirty-six oenological commercial tannins were considered for the study about the effect on copigmentation. Specifically, the following were analyzed: 17 proanthocyanidins comprising 9 procyanidins/prodelphinidins (3 from grapes, 4 from grape seeds and 2 from grape skin) and 8 profisetinidins/prorobinetidins ( 2 from acacia and 6 from quebracho), and 19 hydrolysable tannins comprising 8 gallotannins (4 from nut galls and 4 from tara) and 11 ellagitannins (8 from oak and 3 from chestnut). They were provided by eight different companies: Laffort (Floirac, France), Agrovin (Ciudad Real, Spain), Sofralab (Magenta, France), Institut Oenologique de Champagne (IOC) (Epernay, France), Esseco (Trecate Novara, Italy), AEB (Brescia, Italy), Erblsöh (Geisenheim, Germany) and Vason (Verona, Italy).

Five commercial tannins, representing the main botanical origins, were considered for the study about 
the inhibitory effect on laccase activity. Specifically, three condensed tannins were used: one procyanidin from grape seeds, one procyanidin/prodelphinidin from grape skin and one profisetinidin from quebracho, as well as two hydrolysable tannins : one gallotannin from nut galls and one ellagitannin from oak. All these tannins were provided by Laffort (Floirac, France).

\subsection{Measurement of copigmentation effect}

A model wine solution containing $50 \mathrm{mg} / \mathrm{L}$ of malvidin3-O-monoglucoside was prepared and supplemented with $0.1,0.2$ and $0.4 \mathrm{~g} / \mathrm{L}$ of different commercial tannins using (-)-epicatechin as reference control. These concentrations of tannins correspond to copigment/pigment ratio $(\mathrm{CP} / \mathrm{P})$ of $0,2,4$ and 8 respectively. This study was also developed at three different $\mathrm{pH}(3.1,3.5,3.9)$ and at three different ethanol strength $(10 \%, 12 \%$ and 14\%) in order to determine the influence of $\mathrm{pH}$ and ethanol content on the copigmentation effectiveness of the different oenological tannins. The samples were maintained in airtight conditions. A week later the samples were analyzed.

\subsection{Measurement of the inhibitory effect on Laccase activity}

The inhibition effect of the different type of tannins on laccase activity was measured in a healthy grape juice supplemented with 1.5 units/mL of laccase activity which was also supplemented or not with 20 or $40 \mathrm{~g} / \mathrm{hL}$ of the different tannins. After 10 minutes of contact the laccase activity was determined using the syringaldazine test [9].

\subsection{Evaluation of the protective effect of oenological tannins on the color of white and red wines elaborated with grapes infected with Botrytis cinerea}

Two types of winemaking trials were performed using the grape juices obtained from the healthy and botrytized grapes. The first trial was carried out directly with the white grape juices (white winemaking), whereas the second trial was performed with the white grape juices supplemented with malvidin-3-O-glucoside (pseudo-red winemaking) in order to approach what happens in red winemaking. In both trials the grape juice from healthy grapes was supplemented with a proportion of grape juice from botrytized grapes to reach exactly $1.5 \mathrm{UL} / \mathrm{mL}$ of laccase activity.

\subsection{Color measurements}

The measurement of red color (A520 nm) and CieLab coordinates of the samples used in the experiments of copigmentation and the wines obtained in the experiments with botrytized grapes was done according with Vignault et al. (2019b) [8].

\section{Results and discussion}

\subsection{Effectiveness of the different oenological tannins on copigmentation}

Figure 1 shows, as an example, the influence of the copigment/pigment ratio of one the ellagitannin and
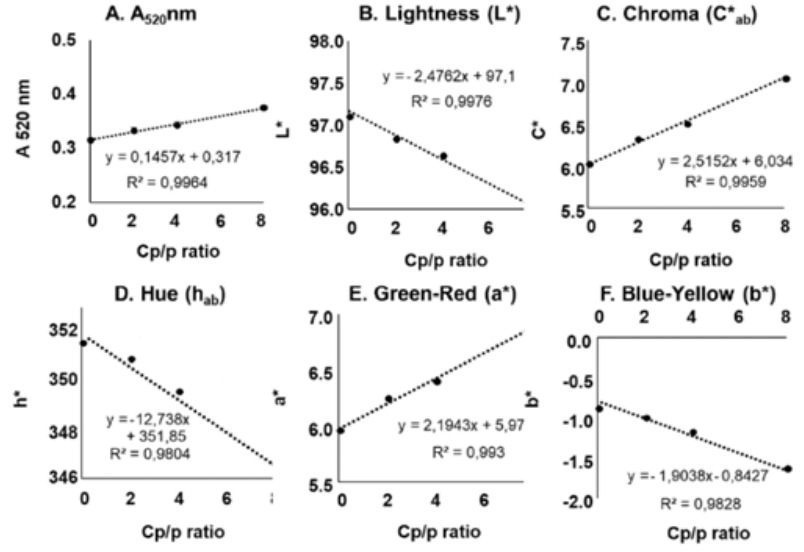

Figure 1. Influence of the Copigment/pigment ratio on the color of malvidin-3-O-glucoside solutions.

malvidin-3-O-glucoside on the red color (A520nm) and the CieLab coordinates.

The values of the slopes indicate the effectiveness of the ellagitannin in improving the color. For A520 nm, C*ab and $\mathrm{a}^{*}$, a higher $\mathrm{Cp}$ indicates a greater impact while for $\mathrm{b}^{*}, \mathrm{~L}^{*}$ and hab, a lower Cp indicates a greater impact. These analyses were performed for all the tannins and the corresponding $\mathrm{Cp}$ are presented in Table 1. The results are very clear and confirm that all types of tannins exert a significant effect on the color of a malvidin-3-O-glucoside solution.

With the aim of better visualize and compare the real copigmentation effectiveness of the different types of tannins a PCA was performed. Figure 2 shows that 3 different clusters are clearly visible: the first containing samples 1 and 2 corresponding to condensed tannins (procyanidins / prodelphinidins - PC/PD and profisetinidins / prorobinetidins - PF/PR), the second comprised mainly of sample 4 (ellagitannins - ET), and the third containing sample 3 (gallotannins - GT).

These results confirm that the botanical origin of oenological tannins influence their effectiveness regarding color stabilization and that gallotannins are the most effective, followed by ellagitannins and condensed tannins.

\subsection{Influence of $\mathrm{pH}$ and ethanol content}

Figure 3 shows the influence of $\mathrm{pH}$ on the copigmentation strength of the different tannins.

The results indicate that epicatechin, seed tannin and skin tannin are more effective as copigments at $\mathrm{pH} 3.1$ whereas quebracho tannins and both hydrolysable tannins are more effective at $\mathrm{pH} 3.5$. In any case all the oenological tannins act as copigments in all the normal wine $\mathrm{pH}$ range.

Figure 4 shows the influence of the ethanol content on the copigmentation strength of the different oenological tannins.

The results epicatechin indicate that epicatechin and all the oenological tannins, with the only exception of quebracho tannins, are more effective copigments when the ethanol content is lower. These results are in accordance with the results obtained previously by Schwarz et al. (2005) [13] who have proved that while the presence of ethanol facilitates anthocyanin and proanthocyanidin extraction, it can disrupt copigmentation complexes. 
Table 1. Copigmentation effectiveness (Cp) of (-)-epicatechin or oenological tannins.
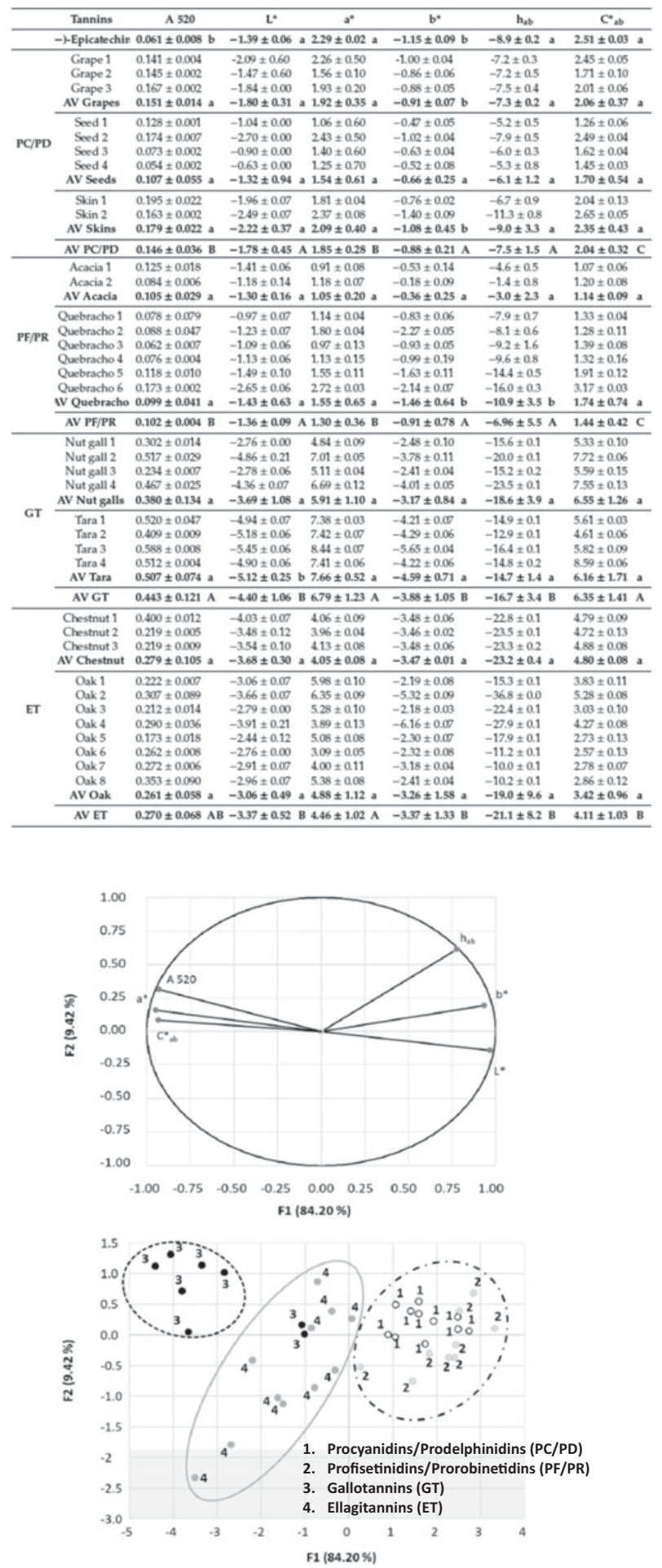

Figure 2. Plot of principal components analysis.

\subsection{Inhibitory effect on Laccase activity and protective effect on wine color}

Table 2 shows the inhibitory effect of the different types of commercial tannins on the laccase activity. All the tannins exerted an inhibitory effect on laccase activity which depended of the tannin dose. Indeed, the higher is the dose used lower is the laccase residual activity. This data confirms the utility of using oenological tannins to

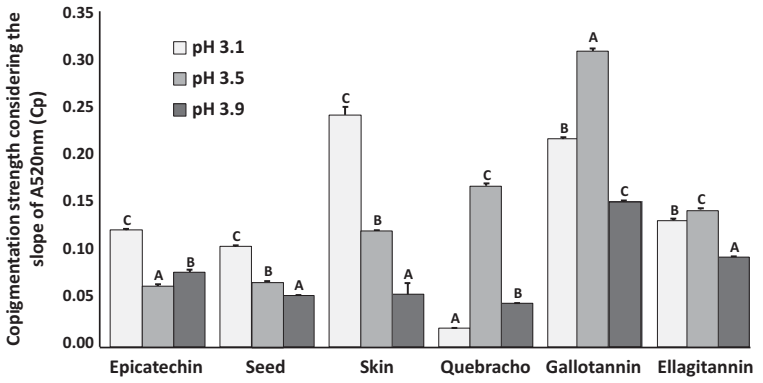

Figure 3. Influence of $\mathrm{pH}$ on copigmentation.

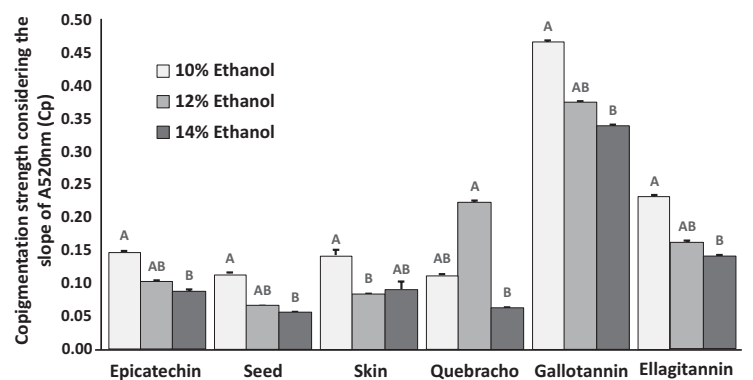

Figure 4. Influence of ethanol content on copigmentation.

Table 2. Inhibitory effect (\%) of the different type of tannins on the laccase activity.

\begin{tabular}{lcc}
\hline \multirow{2}{*}{ Type of tannins } & \multicolumn{2}{c}{ Tannin dose $(\mathrm{g} / \mathrm{hL})$} \\
\cline { 2 - 3 } & 20 & 40 \\
\hline Seed tannin & $23.3 \pm 5.6$ & $31.1 \pm 5.4$ \\
Skin tannin & $24.5 \pm 8.6$ & $31.1 \pm 5.4$ \\
Quebracho Tannin & $20.1 \pm 5.0$ & $36.1 \pm 1.1$ \\
Gallotannin & $21.3 \pm 1.6$ & $34.0 \pm 2.6$ \\
Ellagitannin & $18.5 \pm 7.5$ & $41.5 \pm 3.1$ \\
\hline
\end{tabular}

protect grape juice and wine from browning when grey root is present in the grapes.

All the tannins exerted an inhibitory effect on laccase activity which depended of the tannin dose. Indeed, the higher is the dose used lower is the laccase residual activity. This data confirms the utility of using oenological tannins to protect grape juice and wine from browning when grey root is present in the grapes.

Finally Figs. 5 and 6 show how the supplementation with the different oenological tannins protect the yellow color of white wines and the red color of a pseudo-red wine elaborated with grape juice supplemented with $1.5 \mathrm{UL} / \mathrm{mL}$ of laccase activity purified from grapes seriously infected by Botrytis cinerea.

The results are very clear and confirm that the presence of laccase activity increase yellow color (browning) in white wines and reduce the red color (oxidasic haze) in red wines, and that supplementation with all oenological tannins reduce this negative effects.

\section{Conclusions}

It can be concluded that oenological tannins really exert effect as copigments, being in general more effective hydrolysable tannins than condensed tannins. In addition all the oenological tannins inhibit laccase activity and exert a protective effect on the color of white and red wines. Consequently, it can be asserted that oenological tannins 


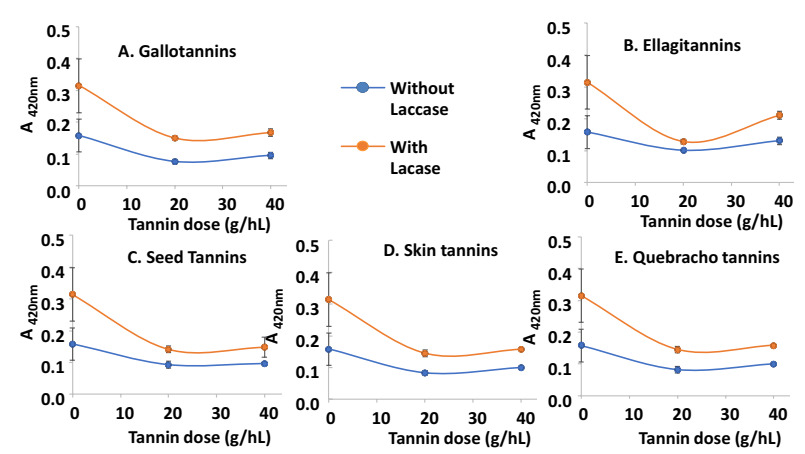

Figure 5. Influence of the supplementation with oenological tannins on the yellow color of white wines from botrytized grapes.

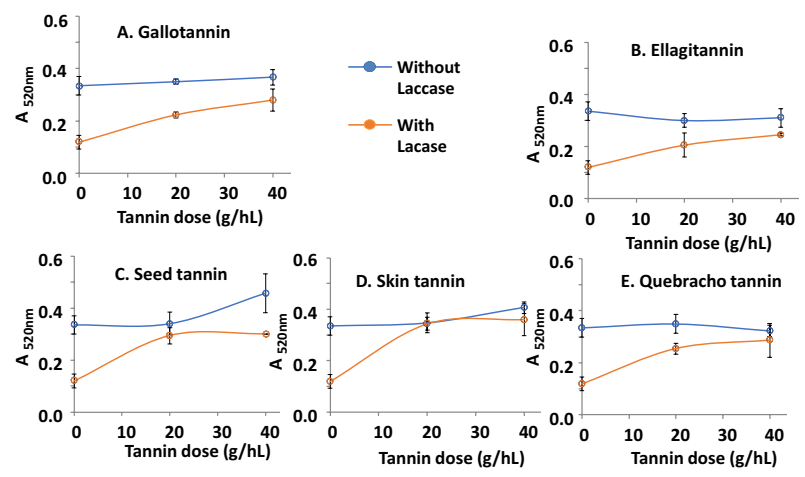

Figure 6. Influence of the supplementation with oenological tannins on the red color of red wines from botrytized grapes.

accomplish with the two new functionalities proposed in the resolutions Oeno-Techno 17-612 [10] and OenoTechno 17-613 [11]. Further studies are required to deep in the knowledge of the functionalities of oenological tannins but the actual findings suggest including these new functionalities of tannins in the OIV International Oenological Codex.

\section{References}

[1] A. Versari, W. du Toit, G.P. Parpinello, Aust. J. Grape Wine Res. 19, 1 (2013)

[2] A.C. Neves, M.I. Spranger, Y. Zhao, M.C. Leandro, B. Sun, J. Agric. Food Chem. 58, 11775 (2010)

[3] A. Vignault, M.R. González-Centeno, O. Pascual, J. Gombau, M. Jourdes, V. Moine, N. Iturmendi, J.M. Canals, F. Zamora, P.L. Teissedre, Food Chem. 268, 210 (2018)

[4] A. Vignault, O. Pascual, J. Gombau, M. Jourdes, V. Moine, M. Fermaud, J. Roudet, J.M. Canals, P.L. Teissedre, F. Zamora, BIO Web of Conferences 12, 02005 (2019)

[5] O. Pascual, A. Vignault, J. Gombau, M. Navarro, S. Gómez-Alonso, E. García-Romero, J. M. Canals, I. Hermosín-Gutíerrez, P.L. Teissedre, F. Zamora, Food Chem. 234, 26 (2017)

[6] A. Vignault, O. Pascual, M. Jourdes, V. Moine, M. Fermaud, J. Roudet, J.M. Canals, P.L. Teissedre, F. Zamora, Oeno One 1, 27 (2019a)

[7] J. Gombau, A. Vignault, O. Pascual, J.M. Canals, P.L. Teissedre, F. Zamora, BIO Web of Conferences 7, 02033 (2016)

[8] A. Vignault, J. Gombau, O. Pascual, M. Jourdes, V. Moine, J.M. Canals, F. Zamora, P.L. Teissedre, Molecules 24, 1448 (2019b)

[9] J. Gombau, A. Vignault, O. Pascual, S. GómezAlonso, E. García-Romero, I. Hermosin, J.M. Canals, P.L. Teissedre, F. Zamora, Oeno One. In Press (2019)

[10] M. Urbano Cuadrado, P.M. Pérez-Juan, M. Luque de Castro, M.A. Gomez-Nieto, Anal. Chim. Acta 553, $99(2005)$

[11] OENO-TECHNO 17-612. "Update to the oenological practice on tannin addition in musts"

[12] OENO-TECHNO 17-613. "Update to the oenological practice on tannin addition in wines"

[13] M. Schwarz, J.J. Picazo-Bacete, P. Winterhalter, I. Hermosín-Gutiérrez, J. Agric. Food Chem. 53, 8372 (2005) 